\title{
Delayed Progression of Cerebral Infarction Despite Neurological Improvement in Unilateral Reversible Cerebral Vasoconstriction
}

\author{
KipyoungJ eon', In-Uk Song', Yong-An Chung', \\ Kijeong Lee ${ }^{1}$ and $\mathrm{J}^{1}$ aseong Koo ${ }^{1 *}$ \\ ${ }^{1}$ Department of Neurology, Catholic University of Korea, \\ Korea \\ ${ }^{2}$ Department of Radiology, Catholic University of Korea, \\ Korea \\ *Corresponding author: J aseong Koo, Department \\ of Neurology, Seoul St. Mary's Hospital, The Catholic \\ University of Korea, 222 Banpo-daero, Seocho-gu, Seoul \\ 06591, Korea
}

Received: July 17, 2017; Accepted: August 11, 2017; Published: August 29, 2017

\begin{abstract}
Previous literatures describing cerebral infarction in Reversible Cerebral Vasoconstriction Syndrome (RCVS) have not address the temporal change of cerebral ischemia in relation to clinical symptoms. A 54-year-old man presented with altered consciousness, aphasia and right hemiparesis. Initially, CT angiography showed diffuse narrowing of left Middle Cerebral Artery (MCA) without any diffusion restriction on brain MRI. Two days after onset, he recovered consciousness and follow-up MRI revealed diffusion restrictions at left frontal and temporal cortices. Five days after onset, aphasia and right hemiparesis improved and MR angiography revealed the restoration of left MCA despite extended lesion at left temporal cortex on T2-weighted MRI. We report the case of a patient with unilateral RCVS who showed initial clinical-MRI mismatch with delayed ischemic change on diffusion-weighted MRI followed by progression of infarction despite neurological improvement. We suggest early augmentation of cerebral blood flow be required in this case.
\end{abstract}

Keywords: Reversible cerebral vasoconstriction syndrome; Cerebral infarction; Clinical-MRI mismatch; Delayed ischemic change

\section{Introduction}

Reversible Cerebral Vasoconstriction Syndrome (RCVS) refers to a condition of acute neurological symptoms with radiological change showing diffuse segmental constrictions of cerebral arteries that resolves spontaneously within some periods [1-4]. Cerebral infarction in RCVS tends to be typically bilateral and watershed in location, reflecting impaired cerebral blood flow due to severe cerebral vasoconstriction [5]. Although there have been previous literatures describing cerebral infarction in patients with RCVS [6,7], the temporal change of cerebral ischemia in relation to clinical symptoms has not been addressed in those literatures. The cerebral ischemia caused by RCVS might be different from that caused by typical cerebral infarction which is accompanied by sudden thrombotic or embolic occlusion of cerebral arteries.

We report the case of a patient with unilateral RCVS who showed initial clinical-MRI mismatch with delayed ischemic change on diffusion-weighted MRI followed by progression of infarction despite restoration of vasoconstriction and neurological improvement.

\section{Case Report}

A 54-year-old man visited Emergency Room (ER) due to altered consciousness. He was a chronic alcoholic and a current smoker. He also had hypertension and dyslipidemia. He was last seen as normal 17 hours before visit to ER but was found to be abnormal 1 hour before. His initial blood pressure was $125 / 77 \mathrm{mmHg}$. The neurological examination revealed decreased consciousness, global aphasia with right hemiplegia (MRC grade I at right arm and leg) and left gaze preponderance (National Institute of Health Stroke Scale (NIHSS) score $=22$ ). The CT angiography, taken 70 minutes after the first abnormal time (FAT), showed multiple segmental constrictions of distal branches and mild diffuse narrowing of M1 portion of left Middle Cerebral Artery (MCA) (Figure 1A).

The MRI, taken 14 hours after the FAT, did not show any diffusionrestricted lesion (Figure 1B and C). The Electroencephalography (EEG) displayed intermittent slow wave in the left frontocentral region without epileptiform discharges. We treated him with intravenous hydration and dual anti-platelets including aspirin and clopidogrel. Two days after onset, he recovered consciousness, but still showed persistent global aphasia with right hemiplegia (MRC grade I at arm

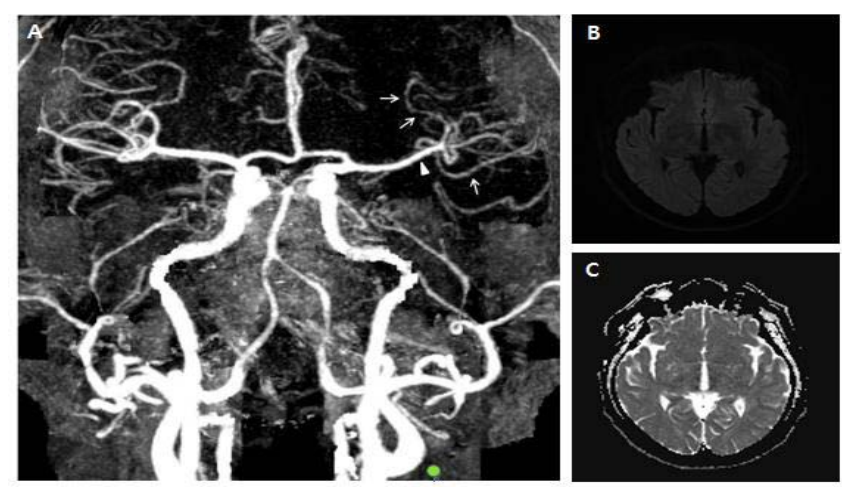

Figure 1: CT angiography and brain MRI at admission. (A) The CT angiography, taken 70 minutes after the FAT showed multiple segmental vasoconstrictions at distal branches (white arrows) and mild diffuse narrowing of M1 portion (white arrowhead). (B, C) The initial brain MRI, taken 14 hours after the FAT, revealed no diffusion-restricted lesion.
Austin J Radiol - Volume 4 Issue 2 - 2017

ISSN : 2473-0637 | www.austinpublishing group.com

Koo et al. (C) All rights are reserved
Citation: Jeon K, Song I-U, Chung Y-A, Lee K and Koo J. Delayed Progression of Cerebral Infarction Despite Neurological Improvement in Unilateral Reversible Cerebral Vasoconstriction. Austin J Radiol. 2017; 4(2): 1070. 

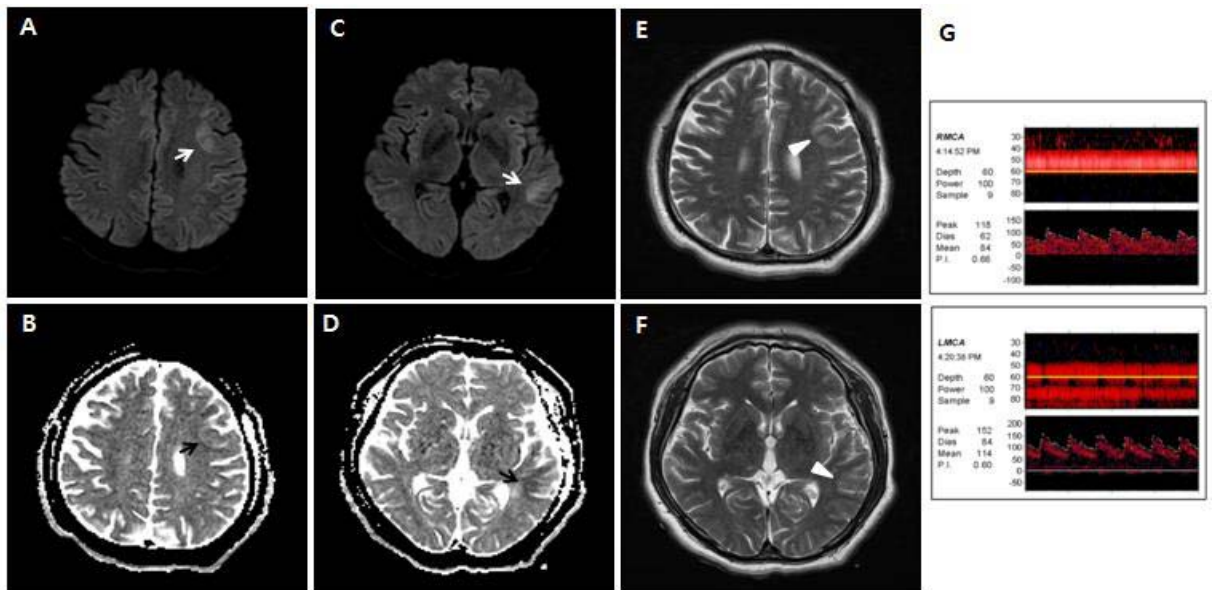

Figure 2: Follow-up brain MRI and Transcranial Doppler Sonography (TCD) taken 2 days after onset. (A-D) The follow-up diffusion-weighted MRI along with ADC map, taken 2 days after onset, revealed delayed ischemic change at the left frontal and temporal cortices (white and black arrows). (E, F) The T2-weighted images on the same day showed high signal intensities restricted to the same regions (white arrowheads). (G) The TCD taken 2 days after onset revealed the increased mean flow velocity at left MCA M1 $(114 \mathrm{~cm} / \mathrm{sec})$.
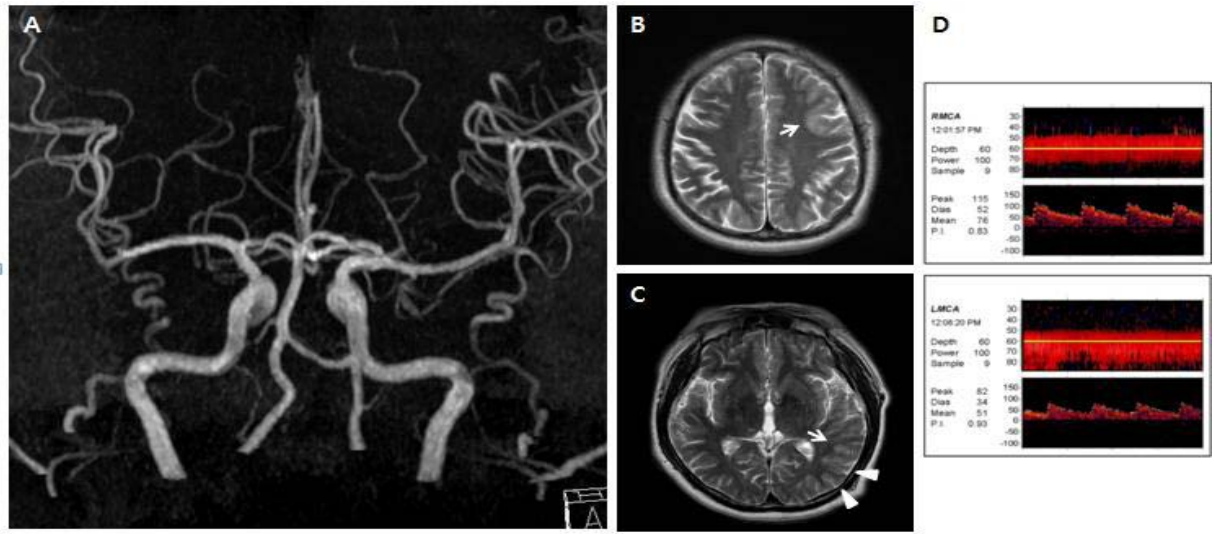

Figure 3: Brain MRI and MR angiography taken 5 days after onset and follow-up TCD about one month later. (A) The MR angiography, taken 5 days after onset, showed the recovery from vasoconstriction at left MCA. (B, C) The follow-up T2-weighted images revealed increased extent of high signal intensity at the left temporal cortex (white arrows: previous lesions, white arrowheads: new lesions). (D) The follow-up TCD about one month later showed the recovery of normal mean flow velocity at left MCA M1 $(51 \mathrm{~cm} / \mathrm{sec})$

and II at leg) (NIHSS score = 13). The follow-up diffusion-weighted and T2-weighted MRI on the same day revealed small patched high signal intensities restricted to left frontal and temporal cortices (Figure 2A-F). The Transcranial Doppler Sonograpy (TCD) showed the increased flow velocity at left MCA (Figure 2G).

On the $5^{\text {th }}$ day after onset, the patient showed motor aphasia and right hemiparesis (MRC grade I at arm and III at leg) (NIHSS score $=11$ ) and the MR angiography revealed the recovery from vasoconstriction at the left MCA (Figure 3A). The T2-weighted MRI on the same day showed increased extent of high signal intensity at the left temporal cortex (Figure 3B and C). Other laboratory evaluations including echocardiography, Holter monitoring and carotid duplex sonography did not show any significant findings. Two weeks after admission, the patient recovered to show mild motor aphasia with right hemiparesis (MRC grade III at arm and III at leg) (NIHSS score =5). The follow-up TCD about 1 month later showed the recovery of normal flow velocity at left MCA (Figure 3D).

\section{Discussion}

Although there are no validated criteria for the diagnosis of RCVS, the key finding is a reversible vasoconstriction. The cerebral vasoconstriction in RCVS begins in small peripheral arterioles and subsequently proceeds centripetally to involve medium and large cerebral artery. The cerebral vasoconstriction in RCVS is characterized by multiple segmental involvements showing beaded appearance and typically resolves spontaneously within days to weeks [2]. Although the patient in this case had atherosclerotic risk factors such as current smoking, hypertension and dyslipidemia, the initial CT angiography showed multiple segmental constrictions of distal branches of left MCA. The mild diffuse narrowing of M1 portion of left MCA may be due to centripetal involvement of vasoconstriction or decreased blood flow to distal branches. The follow-up MR angiography revealed spontaneous improvement of vasoconstriction five days later. The TCD taken two days after onset also showed increased flow velocity at left MCA, which was normalized in the follow-up study. The vasoconstriction in RCVS was typically described as bilateral, but 
unilateral RCVS is also well recognized in previous literatures [8]. RCVS can be caused by various medical conditions including alcohol and can also occur spontaneously [2-4]. In this case, alcohol might be a triggering factor for cerebral vasoconstriction. RCVS is usually accompanied by typical thunderclap headache [2-4]. The patient in this case might have headache, but we could not identify it due to his aphasia. Furthermore, previous literatures showed that about 15\% of RCVS patients expressed non-thunderclap or no headache $[8,9]$. Cardioembolism and intracranial atherosclerosis were excluded by negative findings in appropriate evaluations. Therefore, we concluded that the cerebral infarction in this patient was caused by RCVS although it was atypical in respect of unilateral involvement and relatively rapid resolution of vasoconstriction.

Typical cerebral infarction caused by sudden thrombotic and embolic occlusion of cerebral arteries usually shows diffusion restriction within 12 hours after onset at the latest [10]. In this case, however, the initial MRI taken 14 hours after the FAT showed no diffusion-restricted lesion although the patient showed severe neurological deficits suggesting left MCA territory infarction. Early after onset, diffuse cerebral vasoconstriction may cause only a limited degree of cerebral ischemia, which is enough to cause neuronal dysfunction but insufficient to bring about cytotoxic edema. This phenomenon of clinical-diffusion mismatch has been already known in ischemic penumbra and suggests the degree of ischemia is possibly not sufficient to cause cytotoxic edema but enough to cause neurological deficit by neuronal dysfunction $[11,12]$. The followup MRI, taken 2 days after onset, showed only a limited extent of diffusion restriction at left frontal and temporal cortices despite persistent severe neurological deficits. These findings are suggestive of attenuated but still persistent clinical-diffusion mismatch which is quite unusual in typical thrombotic or embolic cerebral infarction. While the patient was clinically improving and the MR angiography, taken 5 days after onset, showed the recovery from vasoconstriction at left MCA, T2-weighted MRI revealed slightly extended lesion with high signal intensity, especially at the left temporal cortex. This can be interpreted that though cerebral infarction has progressed, the extent of progression was attenuated along with improvement of cerebral blood flow.

The temporal change of MRI, MRA and neurological deficit in our patient has an important therapeutic implication. In the cerebral infarction caused by thrombotic or embolic arterial occlusion, the thrombolytic therapy to restore blood flow has very limited time window. However, in the cerebral ischemia caused by hypoperfusion without sudden arterial occlusion, we can have longer therapeutic time window especially when the patient shows clinical-MRI mismatch. In such a situation, the treatment to improve cerebral perfusion is more necessary than thrombolytic or anti-thrombotic therapy.

\section{Conclusion}

We report the case of a patient with RCVS who showed severe neurological deficit despite no lesion on initial diffusion-weighted MRI. The patient's neurological deficit was improved despite delayed ischemic progression on the follow-up MRI. In a condition of cerebral ischemia caused by hypoperfusion, the progression of cytotoxic neuronal damage can be gradual and we might have more chance to prevent the progression of ischemic injury by improving cerebral perfusion.

\section{Acknowledgement}

We are grateful to Minhye (Ashley) Koo, a student at University of Southern California: Dornsife College of Science, Human Biology major, for her effort and contribution in English editing.

\section{References}

1. Call GK, Fleming MC, Sealfon S, Levine H, Kistler JP, Fisher CM. Reversible cerebral segmental vasoconstriction. Stroke. 1988; 19: 1159-1170.

2. Calabrese LH, Dodick DW, Schwedt TJ, Singhal AB. Narrative review: reversible cerebral vasoconstriction syndromes. Ann Intern Med. 2007; 146: 34-44.

3. Ducros A. Reversible cerebral vasoconstriction syndrome. Lancet Neurol. 2012; 11: 906-917.

4. Miller TR, Shivashankar R, Mossa-Basha M, Gandhi D. Reversible Cerebral Vasoconstriction Syndrome, Part 1: Epidemiology, Pathogenesis, and Clinical Course. AJNR Am J Neuroradiol. 2015; 36: 1392-1399.

5. Miller TR, Shivashankar R, Mossa-Basha M, Gandhi D. Reversible Cerebral Vasoconstriction Syndrome, Part 2: Diagnostic Work-Up, Imaging Evaluation, and Differential Diagnosis. AJNR Am J Neuroradiol. 2015; 36: 1580-1588.

6. Ducros A, Boukobza M, Porcher R, Sarov M, Valade D, Bousser MG. The clinical and radiological spectrum of reversible cerebral vasoconstriction syndrome. A prospective series of 67 patients. Brain. 2007; 130: 3091-3101.

7. Singhal AB, Hajj-Ali RA, Topcuoglu MA, Fok J, Bena J, Yang D, et al. Reversible cerebral vasoconstriction syndromes: analysis of 139 cases. Arch Neurol. 2011; 68: 1005-1012.

8. Wolff V, Armspach JP, Lauer V, Rouyer O, Ducros A, Marescaux C, et al. Ischaemic strokes with reversible vasoconstriction and without thunderclap headache: a variant of the reversible cerebral vasoconstriction syndrome? Cerebrovasc Dis. 2015; 39: 31-38.

9. Wolff V, Ducros A. Reversible Cerebral Vasoconstriction Syndrome without Typical Thunderclap Headache. Headache. 2016; 56: 674-687.

10. Sacco RL, Kasner SE, Broderick JP, Caplan LR, Connors JJ, Culebras A, et al. An updated definition of stroke for the 21st century: a statement for healthcare professionals from the American Heart Association/American Stroke Association. Stroke. 2013; 44: 2064-2089.

11. Hossmann KA. Viability thresholds and the penumbra of focal ischemia. Ann Neurol 1994; 36: 557-565

12. Prosser J, Butcher K, Allport L, Parsons M, MacGregor L, Desmond P, et al. Clinical-diffusion mismatch predicts the putative penumbra with high specificity. Stroke. 2005; 36: 1700-1704.

\footnotetext{
Austin J Radiol - Volume 4 Issue 2 - 2017

ISSN : 2473-0637 | www.austinpublishing group.com

Koo et al. (C) All rights are reserved
}

Citation: Jeon K, Song I-U, Chung Y-A, Lee K and Koo J. Delayed Progression of Cerebral Infarction Despite Neurological Improvement in Unilateral Reversible Cerebral Vasoconstriction. Austin J Radiol. 2017; 4(2): 1070. 